\title{
148. Cortical Projections of the Lateral Thalamic Nuclei in the Cat
}

\author{
By Kahee Nirmi and Hiroyasu Inoshita \\ The Third Department of Anatomy, Okayama University \\ Medical School, Okayama \\ (Comm. by Kô Hirasawa, M. J. A., Sept. 13, 1971)
}

Formerly it was said that the dorsal lateral nucleus has no cortical connections, whereas the posterior lateral nucleus projects fibers to the cerebral cortex. Later studies, however, have demonstrated the cortical projection of both dorsal lateral and posterior lateral nuclei. The present study was undertaken to elucidate the precise organization of cortical projections of these nuclei in the cat by using the Nauta and Gygax ${ }^{3)}$ method.

Material and methods. Unilateral electrolytic lesions were placed in various portions of the left dorsal lateral and posterior lateral nuclei in seven cats with the aid of a stereotaxic instrument under Nembutal anesthesia. In cats 5, 15 and 45 the electrode was inserted vertically through the left cerebral hemisphere to the lateral nuclei of the same side, while in cats $37,42,49$ and 54 it was introduced by a right dorsolateral approach to effect a lesion on the left side. After a survival period of 7 days, the brains were fixed in $10 \%$ neutral formalin for one month, sectioned serially at $40 \mu$ in the transverse plane, and every fourth section was impregnated by the NautaGygax $^{3)}$ method. The terminology here employed followed that of Niimi. ${ }^{5)}$ The posterior lateral nucleus corresponds to the anterior part of the nucleus of the same name of most authors.

Results. In the case with the large lesion involving most of the dorsal lateral nucleus (cat 37), heavy cortical degeneration is found in the middle and posterior suprasylvian gyri, the lower part of the posterolateral gyrus, the anterior and posterior limbic regions, the postsubicular area and the paralimbic region. Milder degeneration is observed in the cortex of the most dorsal portions of the middle and posterior ectosylvian gyri. No degenerated fibers are found in the posterodorsal corner of the posterior suprasylvian gyrus, which belongs to area 19 of Otsuka and Hassler ${ }^{7)}$ (Fig. 1).

The extent of cortical degeneration following localized lesions in the dorsal lateral nucleus is similar to that in cat 37 , but the severely degenerated areas vary with the site of the lesions in this nucleus. In cat 15 having the lesion in the anterior part of the dorsal lateral 
nucleus, heavy degeneration in the cortex is found in the middle suprasylvian gyrus, particularly its anterior and middle portions, and in the middle part of the cingulate gyrus (Fig. 2). In cat 5 with the lesion in the posterior part of the dorsal lateral nucleus, heavy cortical degeneration is noted in the posterior portion of the middle suprasylvian gyrus, the anterior portion of the posterior suprasylvian gyrus, and the posterior and posteroinferior part of the cingulate gyrus (Fig. 3). This suggests strongly that the cortical projection of the dorsal lateral nucleus is organized essentially in a topical manner in the anteroposterior direction, though any part of the dorsal lateral nucleus tends to project fibers diffusely to the entire extent of the cortical projection area of the dorsal lateral nucleus.

In all our cases with lesions in the posterior lateral nucleus, the whole extent of cortical degeneration is similar to that in cases with lesions in the dorsal lateral nucleus. In addition, it includes the anterior suprasylvian gyrus and the anterolateral part of the lateral gyrus (area 19). Heavy degeneration, however, is confined to the

A

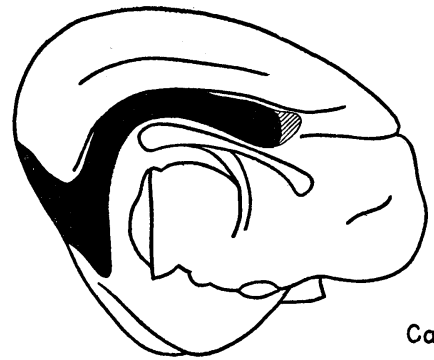

Cat 37
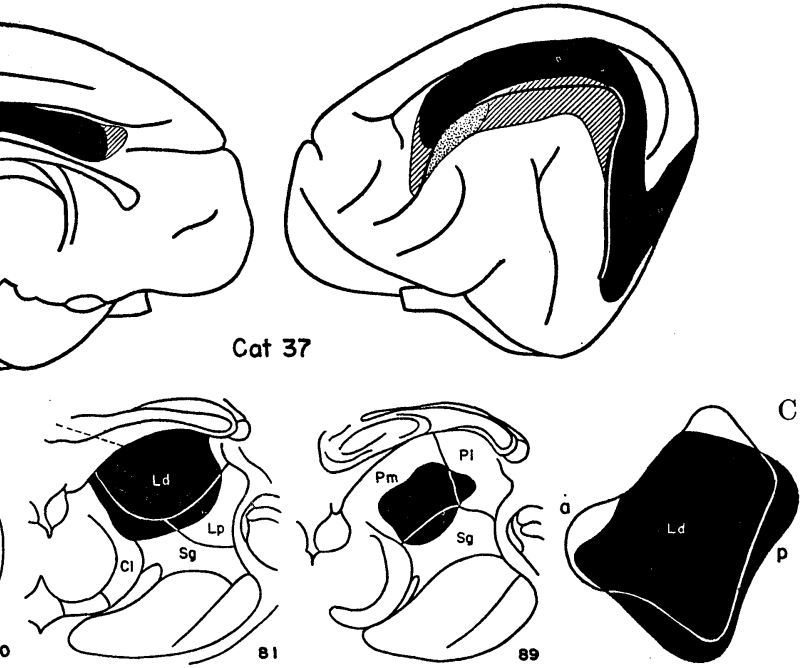

Fig. 1. Showing the extent of the cortical degeneration (A) and the thalamic lesion (B, C) in cat 37 . In this and subsequent figures, heavy degeneration is indicated by solid black, moderate degeneration by oblique hatching and light degeneration by stipple. The lesion (black) is shown on three transverse sections (B) and on an orthogonal reconstruction of the lateral nucleus viewed from lateral (C). The direction of the electrode is indicated by a broken line. a, anterior; $\mathrm{p}$, posterior; $\mathrm{Cl}$, lateral central nucleus; $\mathrm{Ld}$, dorsal lateral nucleus; Lp, posterior lateral nucleus; Pl, lateral pulvinar nucleus; $\mathrm{Pm}$, medial pulvinar nucleus; $\mathrm{Sg}$, suprageniculate nucleus; Vl, lateral ventral nucleus. 
A

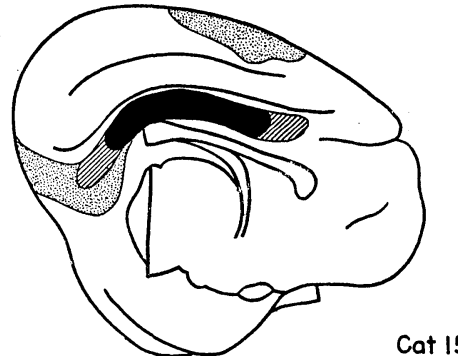

B

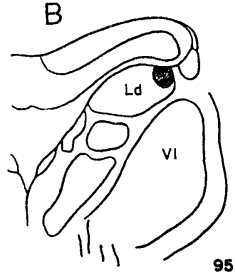

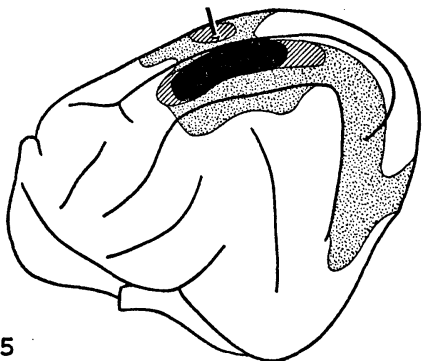

C
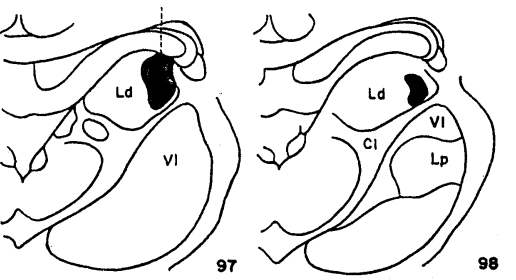

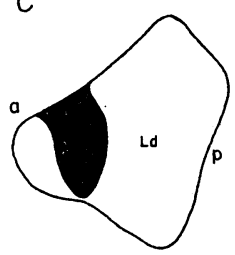

Fig. 2. Showing the lesion $(\mathrm{B}, \mathrm{C})$ and cortical degeneration

(A) in cat 15 .

A

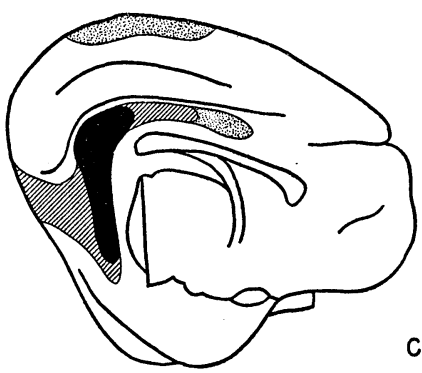

Cat 5
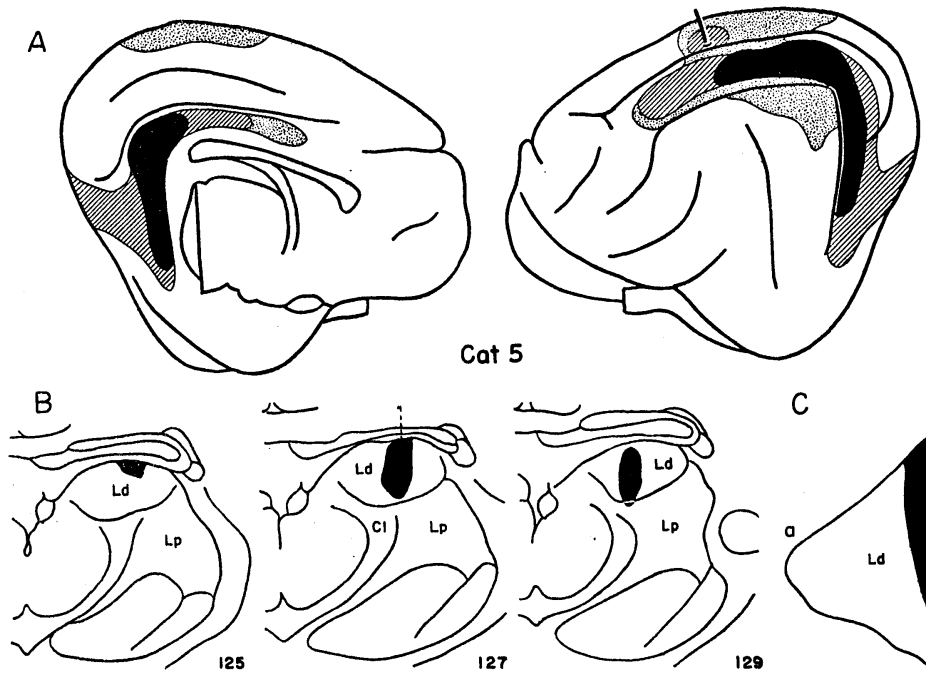

C

Fig. 3. Showing the lesion $(B, C)$ and cortical degeneration (A) in cat 5.

cortex of the anterior and middle suprasylvian gyri, the adjacent part of area 19 in the lateral gyrus, and the cortex around the anterior splenial sulcus, including area 19 in the depth of this sulcus. Within these heavily affected areas, the regions of the most severe degeneration vary with the site of the lesions in the posterior lateral nucleus (Figs. 4-7). In the case with the lesion in the anterior part of the posterior lateral nucleus, the heaviest degeneration in the cortex is 
seen in the middle and posterodorsal parts of the anterior suprasylvian gyrus, the anterior part of the middle suprasylvian gyrus, the lateral part of the lateral gyrus adjacent to the area of degeneration in the suprasplenial gyri (area 19), and the banks and fundus (area 19) of the extreme anterior portion of the splenial sulcus (cat 45). When the lesions are made in the middle part of the posterior lateral nucleus, the heaviest cortical degeneration is found somewhat more caudally
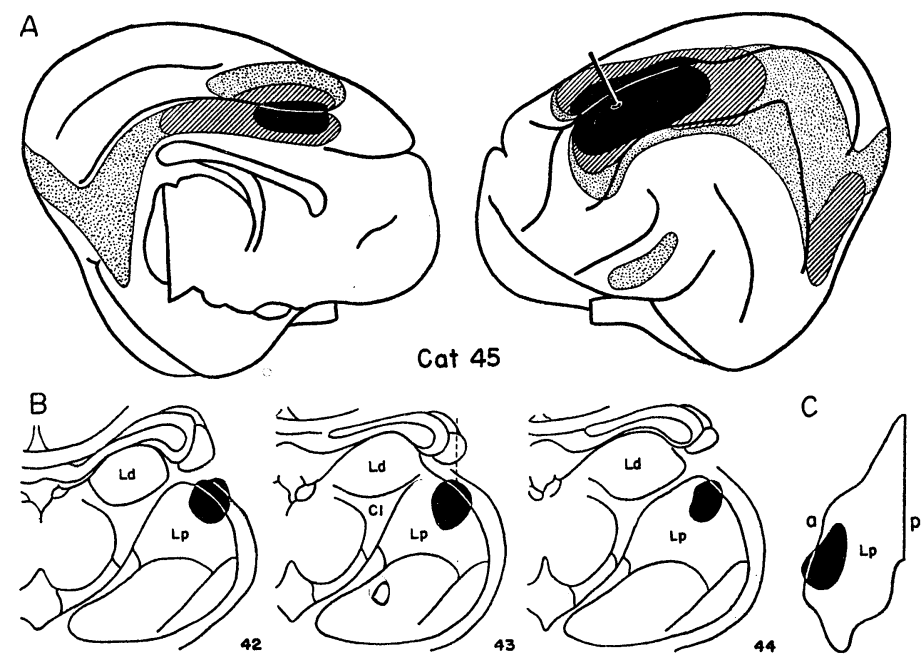

Fig. 4. Showing the lesion $(B, C)$ and cortical degeneration (A) in cat 45 .
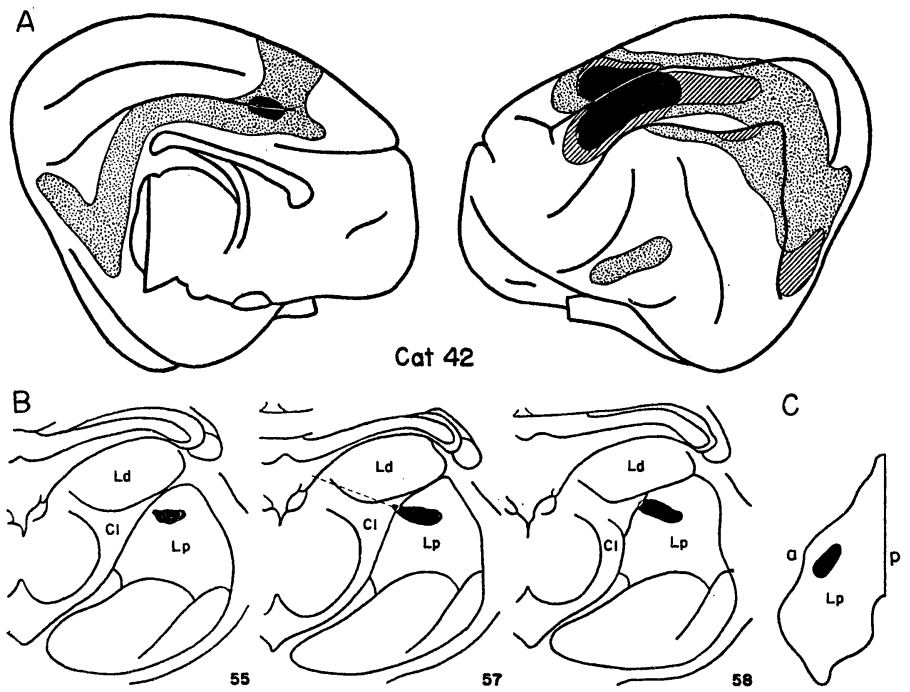

Fig. 5. Showing the lesion (B, C) and cortical degeneration (A) in cat 42 . 
(cats 42 and 54). In the case with the lesion in the posterior part of the posterior lateral nucleus, the heaviest degeneration is present in the cortex of the middle suprasylvian gyrus, the adjoining part of area 19 in the lateral gyrus, and the cortex around the anterior splenial sulcus, including area 19 (cat 49). These data indicate that there is an anteroposterior organization in the principal projection of the posterior lateral nucleus on the cortex. In all our cases with

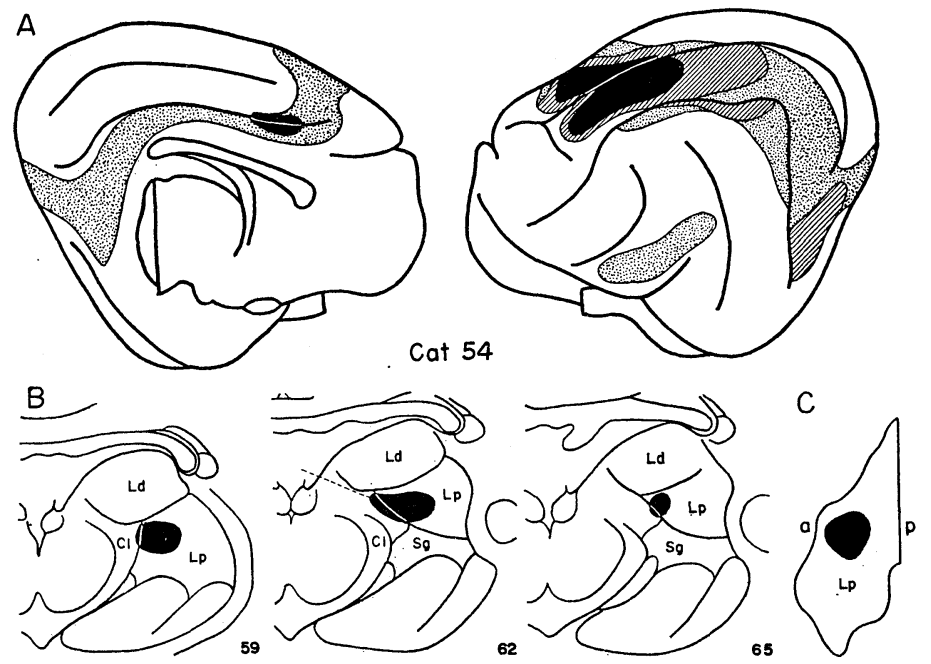

Fig. 6. Showing the lesion (B, C) and cortical degeneration (A) in cat 54.
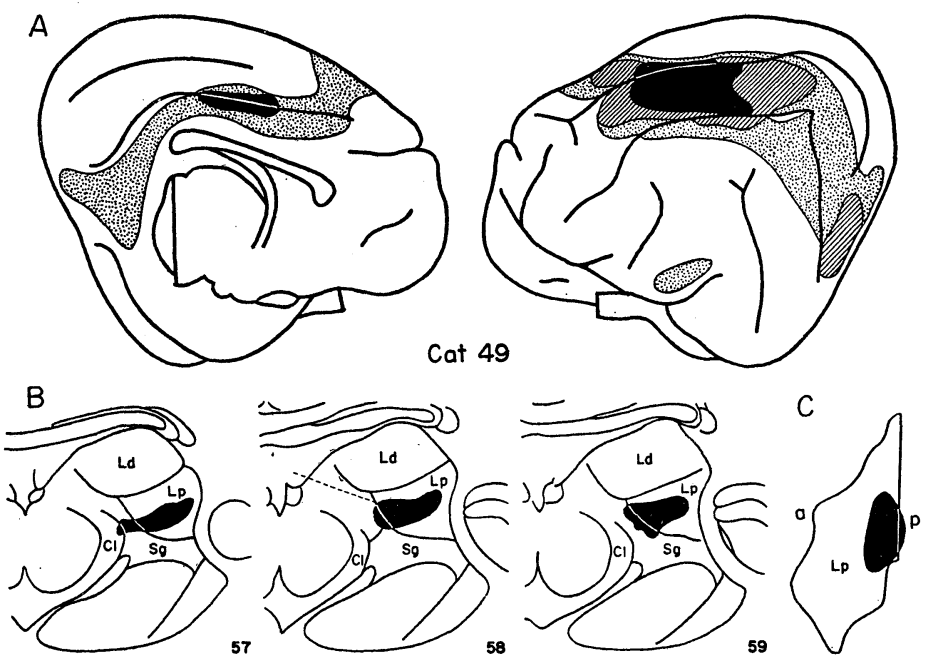

Fig. 7. Showing the lesion (B, C) and cortical degeneration (A) in cat 49 . 
lesions in the posterior lateral nucleus light degeneration is found in the limited cortical part of the anterior sylvian gyrus.

Discussion. Recent investigators demonstrated that the dorsal lateral nucleus of the cat sends fibers to the suprasylvian gyrus (Nauta and Whitlock ${ }^{4)}$ ). Niimi and Sprague ${ }^{6}$ revealed that the dorsal lateral nucleus projects to the anterior and middle suprasylvian gyri, while the posterior lateral nucleus is connected to the anterior, middle and posterior suprasylvian gyri. Locke et al. ${ }^{1)}$ suggested the projection of the dorsal lateral nucleus to the cingulate cortex in the human material, and later demonstrated experimentally that this nucleus sends fibers to the cortex of the posterior cingulate gyrus in the cat (Locke et al. ${ }^{2)}$ ).

Summary. The dorsal lateral nucleus projects fibers diffusely to the cortex of the middle and posterior suprasylvian gyri, the lower part of the posterolateral gyrus, the anterior and posterior limbic regions, the postsubicular area and the paralimbic region. However, the heavy cortical projection of the dorsal lateral nucleus is organized in the anteroposterior direction. The posterior lateral nucleus also projects fibers diffusely to the whole cortical projection area of the dorsal lateral nucleus, as well as to the cortex of the anterior suprasylvian gyrus, the anterolateral part of the lateral gyrus (area 19), and the banks and bottom (area 19) of the splenial sulcus. The heavy cortical projection areas, however, are limited to the anterior and middle suprasylvian gyri, the adjacent part of area 19 in the lateral gyrus, and the cortex around the anterior splenial sulcus, including area 19. Moreover, the heaviest cortical projection of the posterior lateral nucleus to these cortical areas is organized in a topical manner anteroposteriorly.

\section{References}

1) Locke, S., Angevine, J. B., Jr., and Yakovlev, P. I.: Arch. Neur., 4, 355 (1961).

2) Locke, S., Angevine, J. B., Jr., and Yakovlev, P. I.: Arch. Neur., 11, 1 (1964).

3) Nauta, W. J. H., and Gygax, P. A.: Stain Techn., 29, 91 (1954).

4) Nauta, W. J. H., and Whitlock, D. G.: Brain mechanisms and consciousness. Ed. by Delafresnaye. Blackwell, Oxford (1954).

5) Niimi, K.: The thalamus. Anatomy of the brain. Ed. by M. Okamoto and Y. Kusama, Asakura, Tokyo (1971).

6) Niimi, K., and Sprague, J. M.: J. Comp. Neur., 138, 219 (1970).

7) Otsuka, R., and Hassler, R.: Arch. Psychiat. Nervenkr., 203, 212 (1962). 